\title{
Ordre des ingénieurs forestiers du Québec
}

La gestion intégrée des ressources : l'intégration des besoins de la faune dans la planification forestière

e sont plus de 220 personnes qui ont participé, le 31 janvier dernier à Québec, au colloque La gestion intégrée des ressources : l'intégration des besoins de la faune dans la planification forestière. L'événement, organisé par l'Ordre des ingénieurs forestiers du Québec, était parrainé par le ministère du Développement durable, de l'Environnement, de la Faune et des Parcs (MDDEFP), le ministère des Ressources naturelles (MRN) et la Fondation de la faune du Québec. Il visait à permettre la diffusion des résultats des principales initiatives mises en œuvre dans le cadre du programme de Gestion intégrée des ressources pour laménagement durable de la faune en milieu forestier, financé par le MDDEFP et la Fondation.

Les principaux promoteurs de projets, soit les représentants des gestionnaires de Zones d'exploitations contrôlées de la faune sur les terres publiques québécoises, des pourvoyeurs, des trappeurs, des pêcheurs de saumon, des réserves fauniques et de Nature Québec, ainsi que le MRN et le MDDEFP, ont présenté les résultats de leurs travaux respectifs. Qu'il s'agisse de mieux connaitre les sites d'intérêt faunique d'un territoire, les besoins en matière d'habitat pour des espèces importantes comme lorignal, le saumon ou la martre, ou encore de protéger les cours d'eau lors de l'entretien des chemins forestiers, les participants ont été à même de constater que de nombreux outils ont été développés, ou le seront bientôt, afin de mieux intégrer les besoins de la faune et de ses utilisateurs lors de la confection des Plans daménagement forestier intégré (PAFI) des forêts publiques du Québec.

Certaines présentations ont aussi abordé lévaluation des impacts de la prise en considération de la faune sur la possibilité de récolte forestière et sur léconomie des régions. D’autres ont proposé des méthodes permettant de faciliter l'intégration des habitats fauniques dans lapproche d'aménagement écosystémique, lequel cherche à réduire les écarts entre la forêt aménagée et la forêt naturelle.

Les discussions de la plénière ont fait ressortir l'importance de poursuivre les travaux afin détoffer les connaissances de base en matière décologie et d'habitats fauniques et de favoriser une prise en compte plus soutenue des préoccupations des acteurs fauniques dans la planification forestière.

Lactivité sadressait principalement aux professionnels participant de près ou de loin à la production des PAFI. Elle a notamment permis de regrouper un nombre important d'ingénieurs forestiers et de biologistes qui œuvrent au sein des divers organismes concernés par la gestion intégrée des ressources.

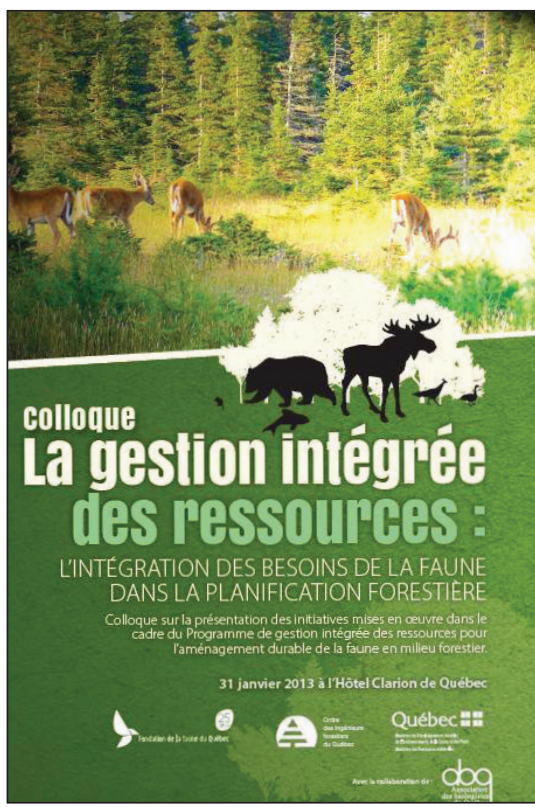

Le 31 janvier 2013 à Québec, I'OIFQ a organisé un colloque entièrement dédié à la gestion intégrée des ressources pour l'aménagement durable de la faune en milieu forestier.

Rappelons que la gestion intégrée des ressources constitue la pierre d'assise du modèle de développement régionalisé mis en place à léchelle du territoire québécois.

Les trois premières années du programme de Gestion intégrée des ressources pour l’aménagement durable de la faune en milieu forestier ont mené à la

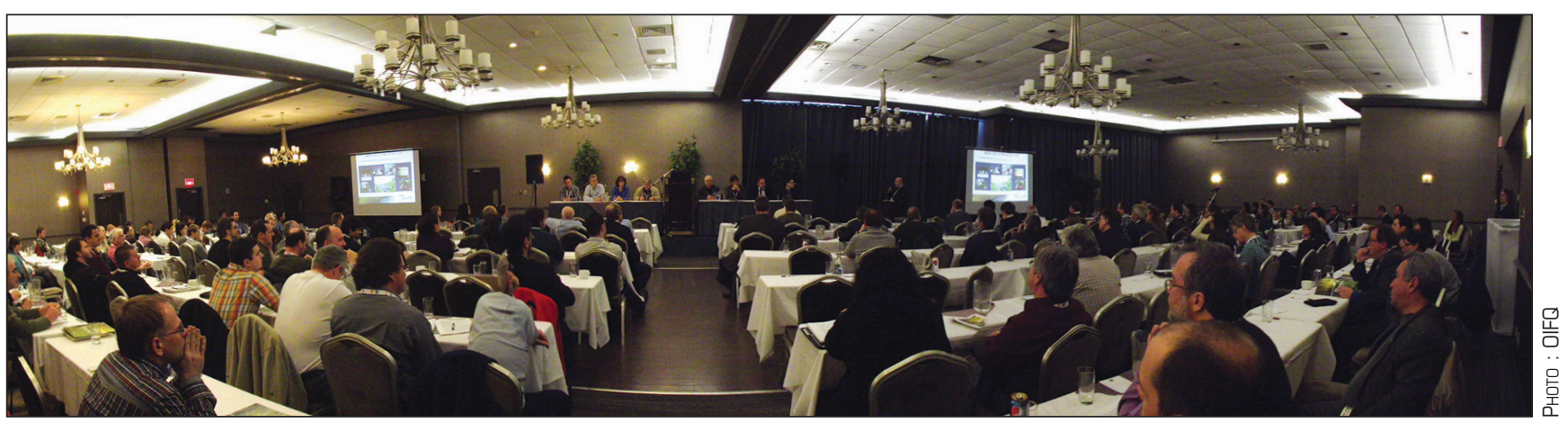

Plus de २२० personnes avaient fait le déplacement pour participer au colloque. 
mise en œuvre d'une soixantaine de projets, en cours ou complétés, dont les résultats méritent une diffusion élargie, notamment dans le contexte de l'adoption des prochains PAFI, prévue pour avril 2013. Ce sont plus de 2 M. \$ qui ont été octroyés à ce jour pour la réalisation des projets. Soulignons que le programme est rendu possible grâce aux revenus générés par la hausse du prix des permis québécois de chasse, de pêche et de piégeage entrée en vigueur le $1^{\mathrm{e}}$ avril 2009. Cette hausse a généré des revenus qui sont réinvestis dans le secteur faunique.

Les présentations faites dans le cadre du colloque sont disponibles sur le site de l'Ordre des ingénieurs forestiers du
Québec à l'adresse : http://www.oifq. com/fr/membres/formation-continue/ formations-passees/colloque-gir-2013/

Pierre Breton, ing.f. Coordonnateur de projets Fondation de la Faune du Québec

\section{Resilience and the Cultural Landscape Understanding and Managing Change in Human-Shaped Environments}

Tobias Plieninger and Claudia Bieling (eds.), 2012. ISBN 978-1-107-02078-8 Cambridge University Press, New York, NY. CAD \$70.95 + shipping. Contact: publicity@cambridge.org

$A^{1}$ ll over the world, efforts are being made to preserve landscapes facing fundamental change as a consequence of widespread agricultural intensification, land abandonment and urbanization. The "cultural landscape" and "resilience" approaches have, until now, been viewed largely as distinct frameworks for understanding the effects of these dynamics and the ways in which they might be adapted or managed. This book brings together these two perspectives, providing new insights into the social-ecological resilience of cultural landscapes by coming to terms with, and challenging, the concepts of driving forces, thresholds, adaptive cycles and adaptive management.

By linking these research communities, this book develops a new perspective on landscape changes. Based on firm conceptual contributions and rich case studies from Europe, the Americas and Australia, it will appeal to anyone interested in analyzing and managing change in human-shaped environments in the context of sustainability.

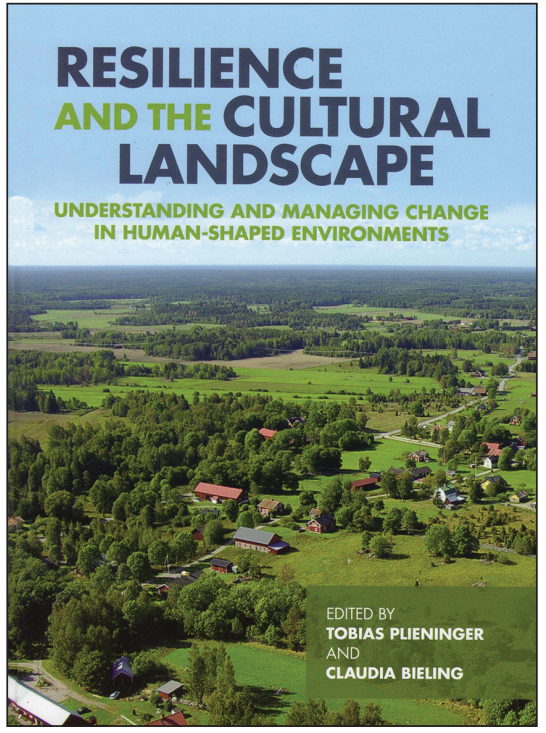

If you purchase a book as the result of a Forestry Chronicle announcement, please mention that you saw it in the Chronicle!

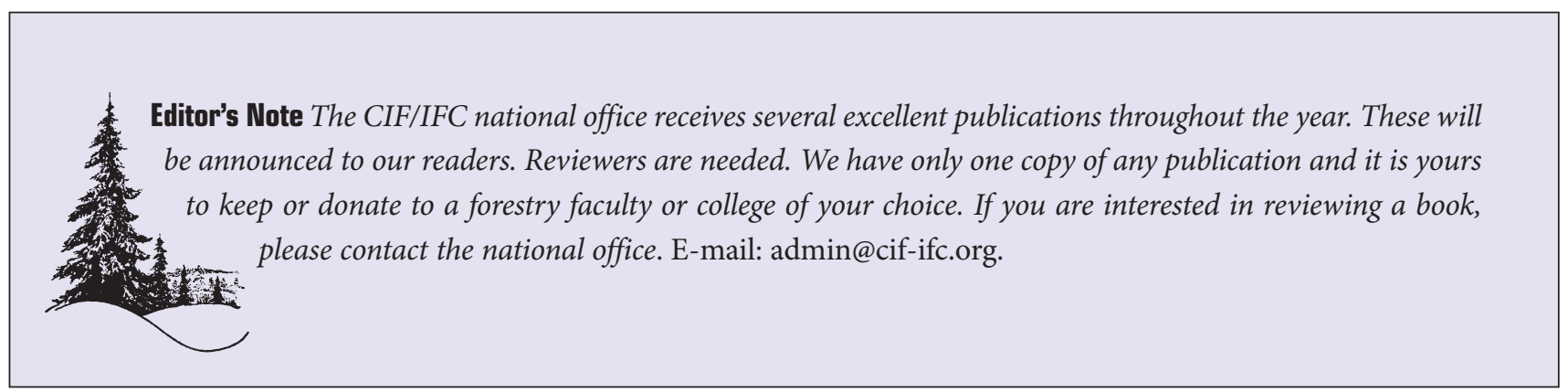

